\title{
ACCESO A LAS TECNOLOGÍAS DE INFORMACIÓN Y LA REDUCCIÓN DE INEQUIDADES*
}

\author{
Nelson Roberto Raudales García, Universidad Nacional Autónoma de Honduras (UNAH), \\ Facultad de Ciencias Económicas, Administrativas y Contables (FCEAC), \\ Instituto de Investigaciones Económicas y Sociales (IIES), \\ Ciudad Universitaria, edificio C2, primer piso, Tel/Fax: 504-22391849 \\ E-mail: nraudales@iies-unah.org
}

\section{RESUMEN}

La época de las Nuevas Tecnologías de información y comunicación (NTIC's) han revolucionado diversos aspectos del diario vivir de los seres humanos, de forma que tales herramientas continúan influenciando en diversas formas en la vida de las personas.

Hoy en día es inevitable el uso de las NTIC's para el desarrollo de actividades médicas, científicas, investigación, innovación, etc. De igual manera, su uso/aplicación en la transferencia de conocimientos no queda separado del desarrollo de actividades orientadas a la escolarización y/o alfabetización, aplicable no solo en las Instituciones de Educación Superior (IES), sino también en instituciones de educación media (EM) y primario.

Asimismo, a nivel operativo tanto de las IES y de EM se vuelve imperante el reto que involucre a su personal docente en desempeñar procesos de transformación y actualización académico-curricular docente, todo ello para lograr aprovechar al máximo el establecimiento y aplicación de medios/políticas educativas que permitan a tales instituciones incursionar eficientemente en la era de las NTIC's. Lo anterior contribuirá además en mejorar tanto el perfil del profesional que egresa de las instituciones de EM como de las IES y les pone en ventaja competitiva y comparativa.

Palabras clave: Nuevas Tecnologías de Información y Comunicación, Competitividad, Investigación y Desarrollo.

*Este trabajo fue seleccionado de trabajos presentados en el Congreso Internacional LACCEI, 2012, en Ciudad de Panamá, Panamá. El documento original ha seguido el proceso de revisión estándar para la Revista Economía y Administración (E\&A), dirigido por el PhD. César H. Ortega J., Director del IIES. 


\title{
ACCESS TO INFORMATION TECHNOLOGY AND REDUCING INEQUALITIES*
}

Vol. 4 $\mathrm{N}^{\circ} 2$ 2013

E\&A

IIES

148

\begin{abstract}
Nelson Roberto Raudales García, Universidad Nacional Autónoma de Honduras (UNAH), Facultad de Ciencias Económicas, Administrativas y Contables (FCEAC), Instituto de Investigaciones Económicas y Sociales (IIES), Ciudad Universitaria, Edificio C2, primer piso, Tel/Fax: 504-22391849 E-mail: nraudales@iies-unah.org
\end{abstract}

\begin{abstract}
The era of the New Information and Communication Technologies (ICT's) have revolutionized many aspects of daily life for humans, so that such tools in various forms continue to influence the lives of people. Today is inevitable the use of ICT's for development of medical, scientific, research, innovation, etc. Similarly, use and application knowledge transfer is not separate / unrelated to the development of education-oriented activities or literacy, applicable not only in Higher Education Institutions (HEIs), but also in educational institutions means (EM) and primary.
\end{abstract}

Also, operating at both the IES and becomes dominant EM challenge involving your staff in performing update processing and teaching academic curriculum, all to achieve maximize the development and implementation of media / political educational institutions such incursions allow efficiently in the era of ICT's. This will also contribute to improve both the professional profile that graduates of institutions EM and HEIs and puts them in competitive and comparative advantage.

Keywords: New Information and Communication Technologies, Research and development.

*This work was selected from papers presented at the International Congress LACCEI, 2012 in Panama City, Panama. The original document has followed the standard review process for the journal Economics and Management (E \& A), led by PhD. Cesar H. J. Ortega, Director of IIES. 


\section{INTRODUCCIÓN}

Desde su aparecimiento e inclusión, las tecnologías de la información y las comunicaciones (TIC'S) se han convertido en herramientas sumamente útiles en la vida del ser humano. Es decir a partir del uso racional de estas TIC's es plausible acceder a conocimientos actualizados que coadyuvan al acceso tanto de información, formación y, transformación de saberes orientados hacia elementos de investigación e innovación.

A partir de los contenidos que continúan, el presente documento se organizan en: Un marco referencial el cual menciona de manera resumida la aplicación y uso de las tecnologías en las diversas disciplinas del conocimiento, es decir orientadas a la trasferencias de saberes actualizados que permiten la formación en sus diversas modalidades de enseñanza. Además el presente incluye de manera sucinta el principal método para descripción del presente, asimismo incluye la parte de descripción de ciertos resultados en cuanto al uso de las tecnologías según quintiles de ingresos, mismo que incluye una comparación a nivel Latinoamericano, además se refleja el porcentaje de uso de las tecnologías por parte de los hogares en ciertos países delárea de América Latina.

Finalmente, el documento incluye ciertas consideraciones generales así como la principal bibliografía utilizada para la elaboración del mismo.

\section{MARCO REFERENCIAL}

Diversos autores han definido aspectos relativos a la educación virtual como medio de apoyo en el proceso enseñanza aprendizaje de las instituciones educativas del nivel medio, primario y de educación superior. En este sentido, (Darin, 2005) define la educación virtual como un conjunto de conocimientos y saberes que se apoyan en soportes, medios tecnológicos que hacen uso de las NTIC's; que permiten que los tutores interactúen con los educandos y con los saberes sin barreras de tiempo, espacio físico, geográfico, etc., para apropiarse de los saberes que permiten su crecimiento académico. Asimismo, a nivel profesional el acceso a las NTIC's permite el desarrollo competitivo de las personas, al lograr en éstas la asimilación y adquisición de conocimientos en la era de la revolución digital que beneficia y potencia los saberes para que los ciudadanos de un estado logren la adquisición de saberes que permitan eficientar el desarrollo académicoprofesional. 
Vol. 4

E\&A

IIES

150

A partir de ello, se puede argumentar que la educación virtual desde los espacios de enseñanza pública y/o privada se desarrolla actividades académico-docentes sin tener necesariamente que disponer de espacios de concurrencia para el desarrollo de tales acometidos. Es por medio del acceso a internet (y específicamente aprovechando el uso de los recursos disponibles para formación/capacitación) que los educandos logran tener accesibilidad a la información que permita su formación, para lograr transformar la realidad socioeconómica de acuerdo a sus problemáticas y/o generar oportunidades que permitan mejoras en cuanto a condiciones de vida y, finalmente les permita la transferencia de conocimientos.

Autores como (Stumpo, et, al, 2013) analizan algunos factores en los cuales describen que desde algunas décadas la vida y desarrollo de los seres humanos están en un periodo de constantes cambios y transición en el cambio de épocas, es decir pasar de una sociedad-empresas desarrollando actividades que la revolución industrial ofrecía.

No obstante, los centros educativos tal como se conciben hoy en día están destinados para preparar académico-profesionalmente a las personas a trascender y ya no desarrollar actividades similares a las permitidas por la revolución industrial, sino más bien actividades de la revolución digital que permiten la era de las NTIC's. Ello debido a que la nueva sociedad basará el desarrollo de actividades más que en la información del conocimiento, por ende éste es saber, es manejar y dosificar apropiadamente la información.

De esto depende la productividad de las organizaciones-empresas las cuales basarán tal productividad en la innovación constante y permanente utilizando cada vez tecnologías más potentes. Por lo tanto, se vuelve necesario desde los diversos agentes económicos, entre ellos el sector académico-educativo preparar a que el educando logre irse adaptando e insertando apropiadamente para ser parte de la revolución digital.

\section{MÉTODOS}

El desarrollo del presente artículo es de naturaleza exploratorio descriptivo y analítico, en donde se caracteriza el uso de las NTIC's como medio para permitir el acceso a medios que incrementen el capital humano, el nivel de conocimientos y con ello permitir la inclusión, reducir las inequidades, promover el crecimiento y desarrollo económico, mejorar el desarrollo social en la vida de las personas, entre 
otras cosas. Para ello, se hace uso de las fuentes de datos de Organismos nacionales (Instituto Nacional de Estadísticas-INE), e internacionales así como de la Comisión Económica para América Latina y el Caribe (CEPAL), instituciones que recolectan sistematizan indicadores de diversa índole para el uso en la investigación. Se hace uso a dichas fuentes por la naturaleza en cuanto al libre acceso de indicadores en los ámbitos pertinentes a la presente investigación.

\section{RESULTADOS}

A continuación se describe los principales resultados encontrados en cuanto al acceso a las NTIC's y como éstas permiten la diferenciación en cuanto acceso a conocimientos que revolucionan el diario vivir de las personas. Para ello se realiza una comparación del nivel de acceso a las comentadas herramientas, posteriormente se menciona tal situación en el caso de Honduras.

\subsection{Comparación a Nivel Latinoamericano}

Latinoamérica presenta asimetrías socioeconómicas diferenciadas entre los países, éstas se manifiestan por sus niveles de inclusión, desarrollo y cohesión social, convivencia de sus ciudadanos, etc. Otra de sus diferencias son expresadas desde la producción o distribución de la riqueza así como a divergencias en cuanto el acceso al uso de la NTIC's entre los países que componen e integran la región (CEPAL, 2011).

Lo anterior muestra que mientras los países desarrollados aumentan en invierten en desarrollo, investigación, e innovación, algunos estados de la región Latinoamericana (o denominados menos avanzados) se han quedado sumamente rezagados en cuanto a la reducción particularmente de la brecha digital como medio de inclusión que permite mejorar entre otros aspectos la competitividad, etc. (Figura No.1)

Un aspecto que llama la atención es la ubicación de Honduras en comparación a los demás países de la región, en el grafico se refleja como dicho país se posiciona entre las naciones con menores índices de desarrollo de las TICS, donde supera apenas a Nicaragua y su indicador se mantiene aún más bajo que el promedio de la región Latinoamericana.

A partir de lo anterior se puede analizar que se vuelve imperante la necesidad de buscar el desarrollo por medio de la implementación de políticas orientadas a

E\&A

IIES

151 
Vol. 4

E\&A

IIES

lograr un mejor acceso alcance y cobertura a las Tecnologías de la información y las comunicaciones, además determinante también la necesidad de fortalecer y contribuir a formar o fomentar el capital social. Es decir, la formación y/o actualización de educadores en los diversos niveles que los sistemas educativos lo requieran con el fin de lograr la transferencia de conocimientos y saberes significativos que permita a los educandos al debida apropiación y/o aplicación en el ámbito que corresponda implementar.

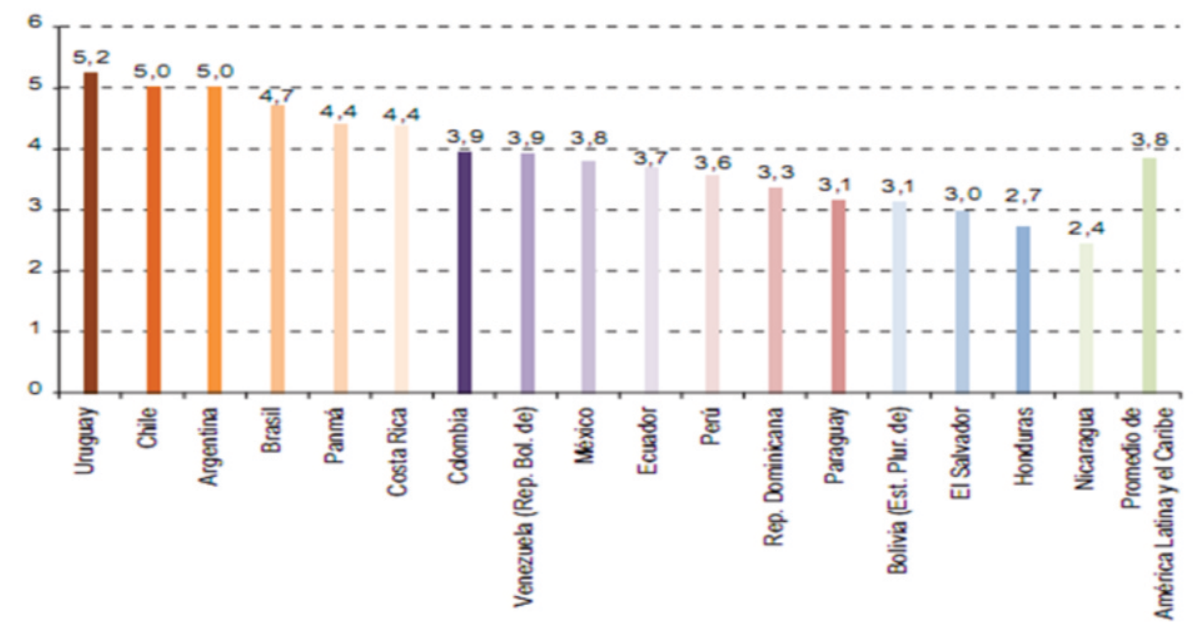

Figura No. 1 América Latina y el Caribe: Índice del desarrollo de las TICS, 2011. (En números índice entre 0 y 10)

Fuente: Comisión Económica para América Latina y el Caribe (CEPAL), sobre la base de Unión Internacional de Telecomunicaciones (UIT), Measuring the Information Society, 2012.

Por otra parte, la difusión o propagación del acceso al Internet en Latinoamérica ha permitido y contribuido a la reducción de las brechas digitales entre los países de la región y los países desarrollados. Se observa la expansión de usuarios al internet como porcentaje de la población en países desarrollados como los países pertenecientes a la Organización para la Cooperación y el Desarrollo Económicos (por sus siglas en inglés OCDE), (OCDE, 2010).

Se puede reflejar como a partir de 2011 en los países de la OCDE el acceso al internet abarcaba el $80 \%$ de la población de dicha región, mientras que para Latinoamérica (Figura No.2) dicha cobertura y acceso apenas llegaba al $40 \%$ de su población (Barrantes, Jordán y Rojas, 2013). 
Los principales resultados encontrados reflejan que a nivel Latinoamericano y específicamente en el caso de Honduras, el acceso a las herramientas de las nuevas tecnologías de información y comunicaciones siguen siendo bajas en comparación con los países desarrollados y los países pertenecientes a la Organización para la Cooperación y el Desarrollo (por sus siglas en inglés OCDE).

De igual manera pudiera considerarse que producto de las desigualdades en el ingreso y el acceso a la educación y las bajas coberturas en cuanto al acceso de servicios públicos que predominan en algunos países de la región condicionan el patrón de acceso al internet por parte de la población con menores recursos. Ello es observable en el caso de algunos países Latinoamericanos, es el caso de Honduras el cual apenas sobrepasa a Nicaragua en el acceso al internet y donde su nivel de acceso se muestra sumamente más bajo que el promedio de la región Latinoamericana. (CEPAL, 2010).

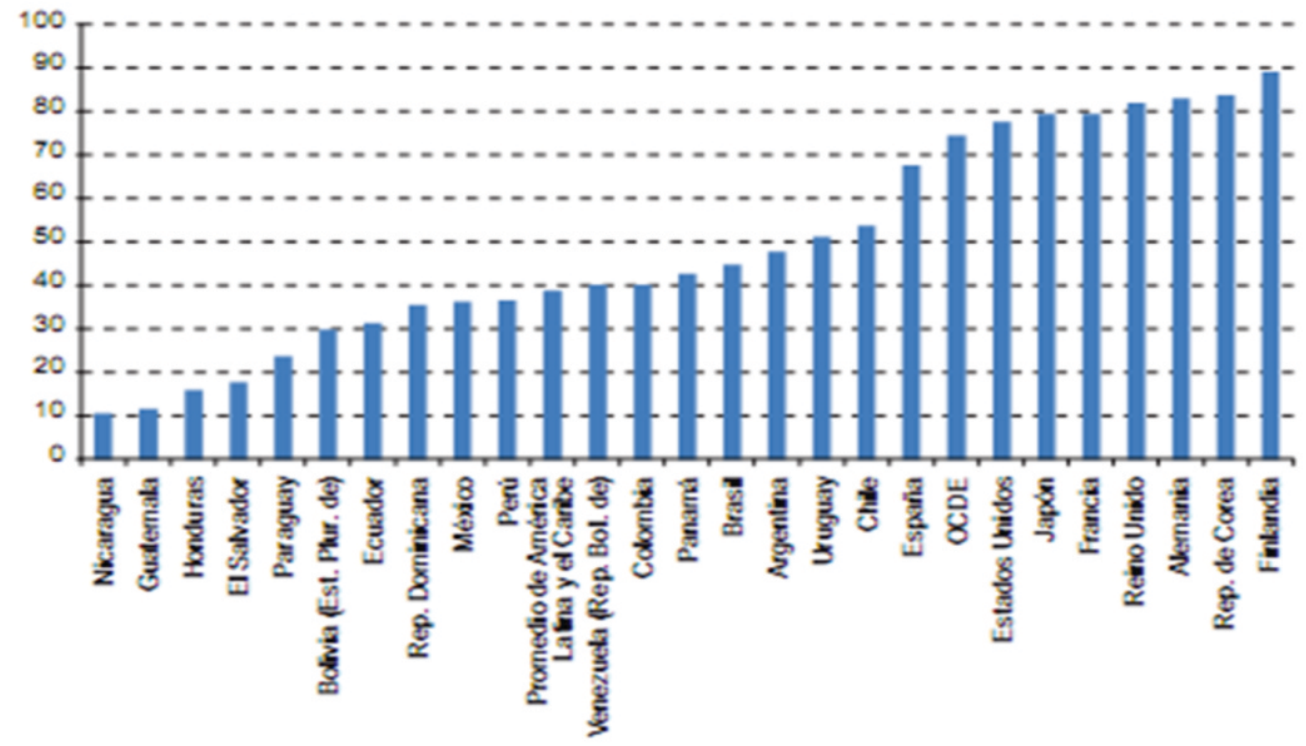

Figura No. 2 Penetración de usuarios al internet, 2011 (en \%)

Fuente: Comisión Económica para América Latina y el Caribe (CEPAL), sobre la base de Unión Internacional de Telecomunicaciones (UIT), Measuring the Information Society, 2012. 
Vol. 4

E\&A

IIES

154

Además, el acceso al internet muestra diferencias en la población según estratos económicos, al observar dicho acceso puede reflejarse como el quintil de la población que obtiene mayores ingresos (quintil 5) muestra una tasa de uso que se quintuplica en comparación con el estrato económico de menores ingresos (quintil 1). Al observar la Figura No. 3 se puede reflejar que aproximadamente del quintil 5 , tienen tasa de uso de $60 \%$ aproximadamente, mientras que la población del quintil 1 dicho porcentaje de acceso llega apenas al 11\% aproximadamente.

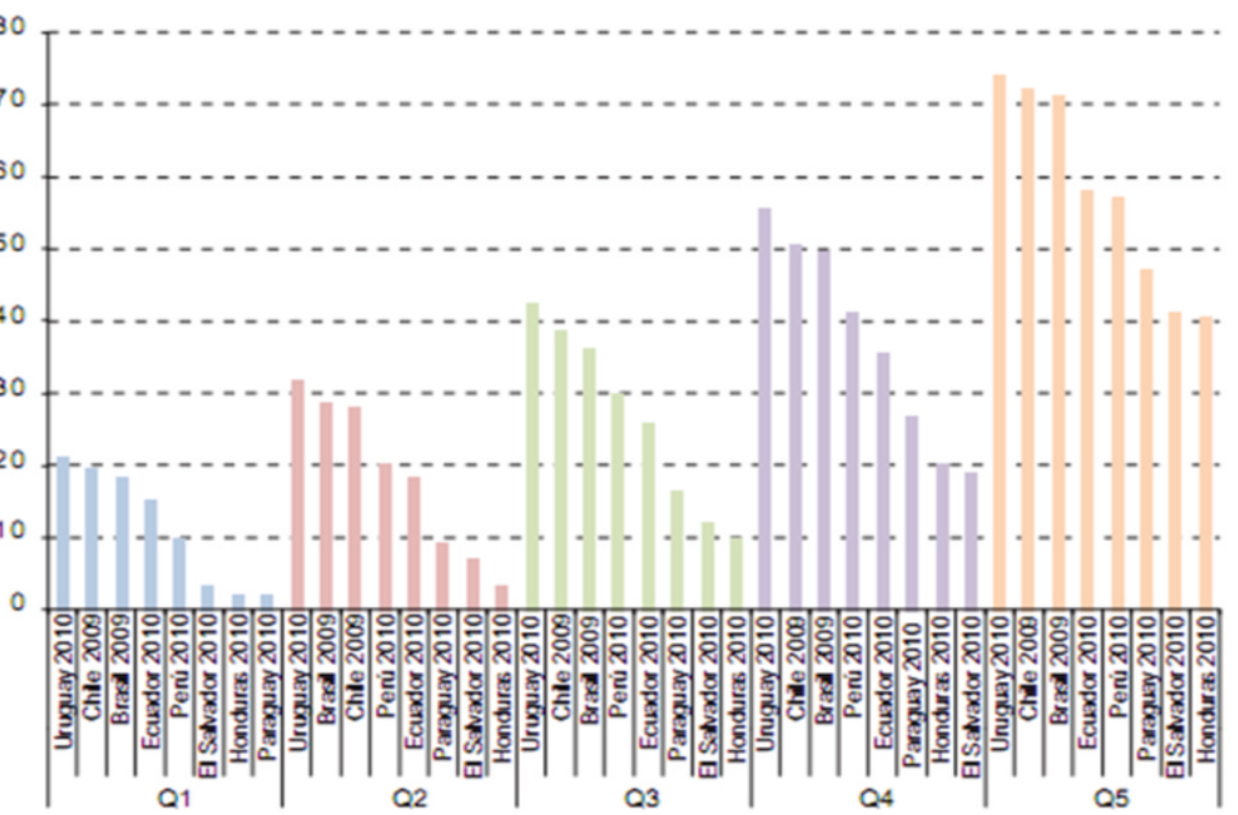

Figura No. 3. Uso de Internet según quintiles de ingresos (en \%)

Fuente: Comisión Económica para América Latina y el Caribe (CEPAL), sobre la base de Observatorio para la Sociedad de la Información en Latinoamérica y el Caribe (OSILAC), con base en información de encuestas de hogares de los institutos nacionales de estadísticas.

Por otra parte, el indicador de acceso a las NTIC's en los hogares se ve diferenciada por área de residencia. Es decir, se sabe de mayores niveles de desarrollo, acceso a bienes, servicios y condiciones de acceso que permiten disponen las áreas urbanas que las hacen más atractivas en comparación con las áreas rurales, desde esta dimensión geográfica se puede observar la diferencia en cuanto al acceso de esta herramienta de suma importancia que conecta e interconecta a los ciudadanos al mundo globalizado. En la Figura No.4 se observa el porcentaje de hogares con acceso diferenciado el tipo de localización (urbano/rural). 


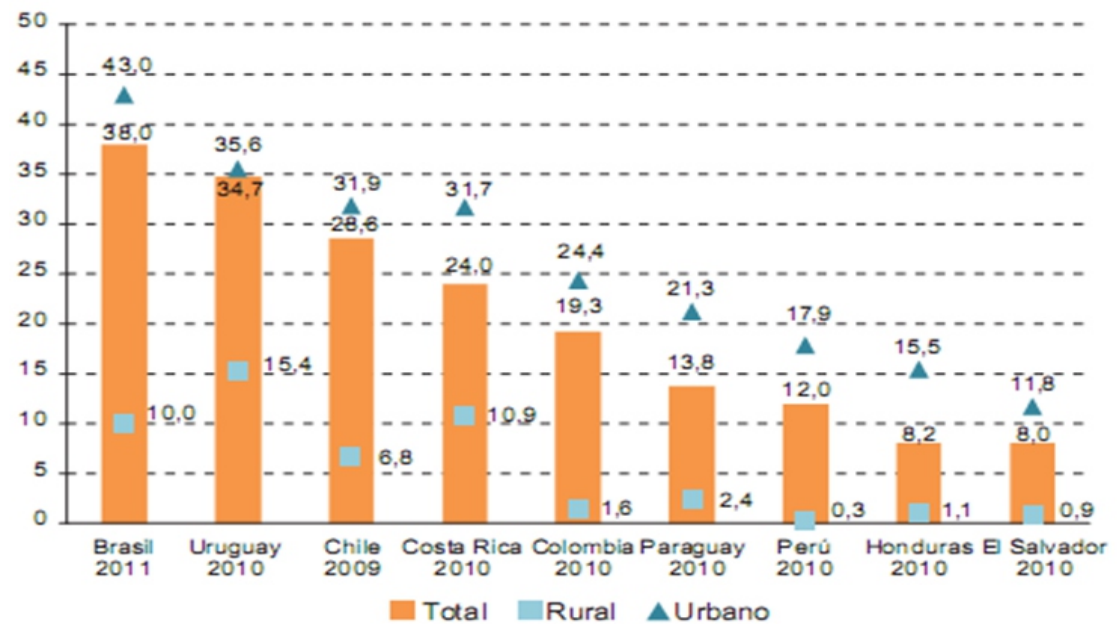

Figura No. 4 Hogares con acceso a internet según área de residencia y a nivel nacional (en \%)

Fuente: Comisión Económica para América Latina y el Caribe (CEPAL), sobre la base de Observatorio para la Sociedad de la Información en Latinoamérica y el Caribe (OSILAC), con base en información de encuestas de hogares de los institutos nacionales de estadísticas.

Por otra parte se ha podido observar con el auge e implementación de las NTIC's en las políticas públicas de los países desarrollados en el mundo. Esto con el propósito de generar beneficios y oportunidades que genera el uso adecuado así como la implementación de nuevas formas de enseñanza que permitan acelerar y realizar una transición eficiente hacia la sociedad de la información (Hilbert, Bustos y Ferraz, 2005, mencionado en CEPAL, 2003).

Tal transición debe estar impulsada por los cambios por los nuevos medios que se dispone para crear, compartir, transferir, comunicar, divulgar, etc., conocimientos que bien aprovechados logren convertirse en información mediante el uso de las tecnologías digitales. Todo este cumulo de insumos de información hoy en día se han visto aprovechados al máximo en ciertos sectores de las sociedades desarrolladas, transición que se refleja en la aparición de nuevas formas de organización social y productiva (CEPAL, 2003).

Asimismo, los países de la región que logren apropiarse y empoderarse del auge tecnológico actual contribuirán con sus sociedades en brindarles oportunidades reales y promisorias, brindando oportunidades globalizantes tangibles que en décadas pasadas no se había logrado. 
Vol. 4

E\&A

IIES

156

Esta oportunidad debido a la magnitud del cambio tecnológico con el que los ciudadanos de las sociedades digitales se enfrentan, esto permitirá un abanico de oportunidades y grado de conciencia que los países pueden aprovechar para recibir las recompensas del cambio.

No obstante, también es posible que, en el largo recuento histórico no haya existido una puerta hacia el progreso y desarrollo como se ha observado en esta época de modernidad, lo cual puede significar un riesgo de desestimar cierta oportunidad que añoren las generaciones venideras (CEPAL, 2003).

Por otra parte, se ha reflejado que los países que no disponen de políticas orientadas a mejorar el acceso, a la cobertura y calidad de una vía apropiada para acceder al internet (Figura No. 5). Se estancan en cuanto a su crecimiento económico, a nivel educativo se restringen los avances recientes de diversos temas, etc., y por consiguiente tales países se mantienen en la cola del desarrollo.

Ante ello se puede observar comparativamente a Honduras, el cual se ubica como en ciertos indicadores de desarrollo, en último lugar en cuanto a la definición de políticas de TIC en la educación. El no disponer de tales medidas regulatorias que contribuyan a brindar una educación de mejor nivel, calidad, acceso y, con ello reducir la cobertura, permitiría al país en ubicarse en mejores posiciones en cuanto a la medición de ciertos indicadores sociales, económicos y de diversa índole.

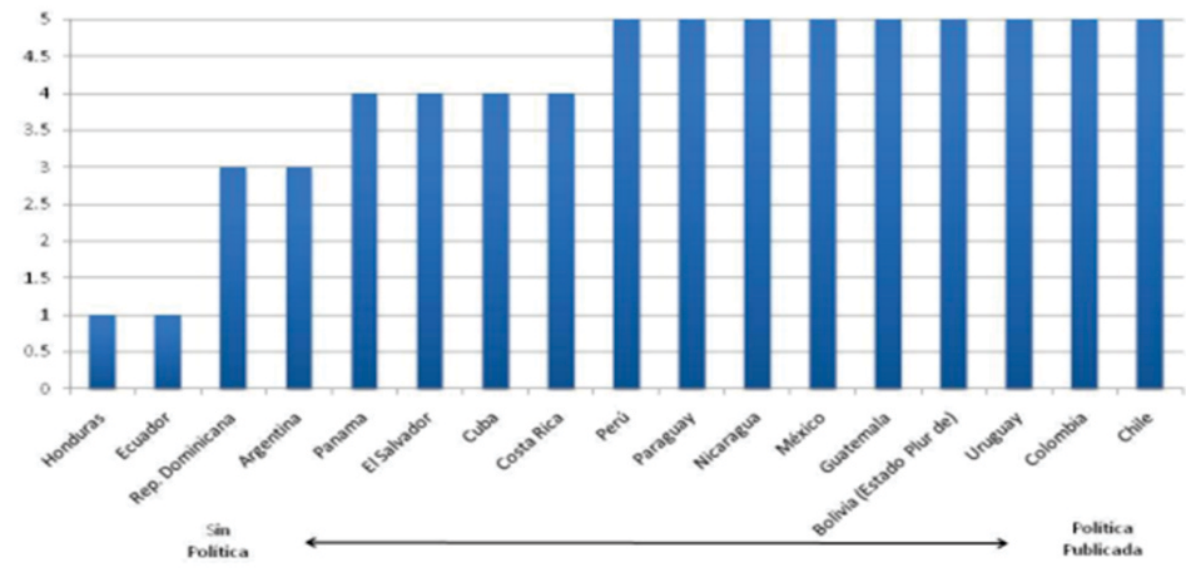

Figura No. 5. América Latina (17 países), grado de formalización de la política de TIC en educación

Fuente: Trucco, D. (2011). "Las Tic para la educación en américa latina y el caribe. Riesgos y oportunidades", Buenos Aires, Argentina. 


\section{CONSIDERACIONES GENERALES}

A partir de las concepciones teóricas definidas previamente en relación a la importancia de las NTIC's, se ha reflejado que están se vuelven importantes para la vida cotidiana en el desarrollo de actividades de las personas.

Asimismo, el uso de estas herramientas ha servido de soporte para el desarrollo de la educación como un conjunto de conocimientos y saberes que permiten interactuar, rompiendo las barreras de espacios geográficos a los agentes del sistema educativo de los países. Lógicamente, deben existir las herramientas tecnológicas, logísticas, infraestructuras apropiadas, etc., y, de diversa índole que permita la apropiación e implementación de estas NTIC's en el conjunto de actividades del ser humano, aún en el ámbito académico.

Por otra parte, se ha reflejado que la apropiación, uso y/o aplicación racional de las NITC's han contribuido a mejorar la productividad de los diversos agentes económicos en diversos ámbitos, implementar procesos de innovación en los espacios público-privados.

No obstante, se ha observado que existen divergencias socioeconómicas de diversa índole entre países desarrollados y los denominados en vías de desarrollo, se puede reflejar también en cuanto a diferencias en relación al acceso y uso de las NTIC's como medio o forma para acceder a las sociedades encontrar oportunidades y retos que permitan a sus ciudadanos disponer de niveles de inclusión, cohesión social, desarrollo económico y social, convivencia entre sus ciudadanos lo cual permitirá su inclusión en la vida productiva del entorno económico y por ende esto contribuirá en el corto y mediano plazo a tener condiciones de vida decorosas.

De los resultados observados se ha podido vislumbrar, como mientras los países desarrollados continúan en un proceso de aumento de inversión en aspectos relacionados a investigación y desarrollo que contribuye a mantener niveles de competitiva empresarial, no sucede así con ciertos países de la Región Latinoamericana los cuales al igual que Honduras se han quedado rezagados en cuanto a la reducción de las brechas digitales para lograr la competitividad.

Asimismo, se ha vislumbrado en los indicadores Latinoamericanos que para el año 2011, Honduras supera apenas a Nicaragua en cuanto al índice del desarrollo de las NTIC's y dicho indicador se mantiene en niveles relativamente más bajos que 
Vol. 4

E\&A

IIES

158

el promedio de la región Latinoamericana.

Otro de los aspectos de rezago digital de la región es la propagación o difusión y acceso al internet, mientras para el año 2011 en países de la OCDE el acceso y cobertura abarcaba el $80 \%$ de su población de dicha región, no así la región Latinoamericana donde dicho porcentaje llega a la mitad de cobertura de la población de esta zona geográfica.

Producto de dicha rezago digital se debe particularmente a problemas estructurales entre ellas las desigualdades en cuanto al ingreso, inequidades sociales y acceso a educación así como a las bajas tasas de cobertura de servicios públicos predominantes en los países de la región.

De igual forma dicha desventaja es manifiesta si se observa por estratos económicos, donde notoriamente se observa como el quintil cinco (5) o estrato socioeconómico alto la tasa de acceso se quintuplica en comparación con el quintil uno (1), que significa el estrato con ingresos más bajos de la población; Dichas tasas de acceso llegan al $60 \%$ y $11 \%$ aproximadamente respectivamente.

Otro de los hallazgos relevantes es que si bien es cierto el acceso al internet es más propenso o de mayor acceso a nivel de los estratos socioeconómicos altos en comparación con los sectores pobres.

También es diferenciado dicho acceso si se observa por zonas de residencia, es decir se reflejan mayores tasas de acceso en el área urbana que en comparación con el área rural de los países seleccionados de la región Latinoamericana. En el caso de Honduras, el acceso a nivel urbano supera enormemente a las zonas rurales; asimismo Honduras apenas supera a El Salvador en cuanto a tasas de acceso por áreas geográficas.

Asimismo, se ha podido reflejar como la implementación de políticas públicas orientadas a lograr el acceso a las NTIC's genera beneficios plausibles y oportunidades de nuevas formas de enseñanza que contribuyen en acelerar una transición hacia la sociedad de la información.

Ello permitirá a los países de la región diferenciarse competitivamente para aprovechar al máximo las oportunidades que los fenómenos globalizantes brindan a las sociedades cada vez más capacitadas y con mejores medios de acceso a 
formaciones y apropiación de nuevos saberes que en décadas anteriores no se reflejaban.

Puede notarse como patrón de comportamiento en cuanto a los indicadores de acceso a la NTIC's de los países de la Región Latinoamericana, que mientras los pobladores ubicados en el quintil más alto de ingresos logran tener coberturas sumamente altas las cuales quintuplican el acceso a las tecnologías de acceso en comparación con la población que se encuentra en el quintil más bajo.

Consecuentemente, a los bajos niveles de acceso al internet por parte de la población ubicada en el quintil más bajo de ingresos, también se combina los indicadores de acceso a las tecnologías de las poblaciones ubicadas en las área rurales.

A partir de ello, se refleja que a nivel Latinoamericano el promedio de acceso a las NTIC's en estas últimas áreas se refleja sumamente distanciada de los indicadores de acceso en comparación con el área urbana y, como es un patrón invariable Honduras se ubica en la penúltima posición de acceso a las NTIC's, superando apenas a El Salvador para el año 2011.

E\&A

IIES

159

A partir de la descripción y análisis de indicadores descritos previamente, se ha reflejado que los países que no disponen de políticas orientadas a mejorar el acceso, a la cobertura y calidad de una vía apropiada para acceder al internet se estancan en cuanto a su crecimiento económico, a nivel educativo se restringen los avances recientes de diversos temas, etc., y por consiguiente tales países se mantienen en la cola del desarrollo y, a partir de la no implementación o definición de políticas de NTIC's, se ha reflejado como Honduras se queda en la última posición en cuanto a la comparación a nivel Latinoamericano para el año 2011.

Por lo tanto la definición se puede comentar o reflexionar que se vuelve imperante la definición de políticas de TIC y educación en la región, ya que permiten a los países que han implementado y aplicado en el ámbito educativo dichas políticas, retribuciones que permitirían mejorar en cuanto a posiciones de sus indicadores en el ámbito internacional. 
Vol. 4

E\&A

IIES

160

\section{BIBLIOGRAFÍA}

Barrantes, Jordán y Rojas. (2013). Mencionado en CEPAL, 2012. "Economía digital para el cambio estructural y desigualdad. Santiago de Chile.

CEPAL. (2011). "Panorama Económico y Social de América Latina y el Caribe, 2010" Santiago de Chile.

CEPAL. (2012). "Measuring the Information Society, 2012." Sobre la base de Unión Internacional de Telecomunicaciones (UIT). Santiago de Chile.

CEPAL. (2003). "Tecnologías de la información y la comunicación para la educación en contextos rurales en América Latina y el Caribe: cerrando brechas", documento elaborado por el componente educación del programa@LIS2 a solicitud del Gobierno de Colombia como aporte al proceso de negociación de la VI Cumbre de las Américas. Santiago de Chile.

CEPAL. (2010). "Panorama Económico y Social de América Latina y el Caribe, 2010" Santiago de Chile.

Darin, S. (2005). "Educación virtual" (1 ${ }^{\mathrm{a}}$ parte), recuperado el 5-09-2012 desde http://caeti.uai.edu.ar/boletin/03/05/01/36.asp

OCDE (Organización de Cooperación y Desarrollo Económico (2010). Are the New Millennium Learners Making the Grade? Technology Use and Educational Performance in PISA, 2006. Paris, Francia.

Stumpo, et, al, (2013). Entre mitos y realidades, TIC, políticas públicas y desarrollo productivo en América Latina. CEPAL, Santiago de Chile.

Trucco, D. (2011). "Las tic para la educación en América Latina y el Caribe. Riesgos y oportunidades". Buenos Aires, Argentina. 